\title{
Entropy generation in a channel resembling gas turbine cooling passage: Effect of rotation number and density ratio on entropy generation
}

\author{
M BASHA, M AL-QAHTANI and B S YILBAS* \\ Mechanical Engineering Department, King Fahd University of Petroleum and \\ Minerals (KFUPM), Dhahran 31261, Saudi Arabia \\ e-mail: \{bsyilbas; mqahtani; nbbasha\}@kfupm.edu.sa
}

MS received 21 October 2007; revised 6 May 2009

\begin{abstract}
Flow into a passage resembling a gas turbine blade cooling passage is considered and entropy generation rate in the passage is examined for unique rotation number and density ratios. In the simulations, leading and trailing walls of the passage are assumed to be at constant temperature. A control volume approach is introduced to discretize the governing equations of flow, heat transfer, and entropy generation. Reynolds stress turbulence model is accommodated in the simulation to account for the turbulence. The study is extended to include two rotational speeds and three density ratios. The passage aspect ratio is kept 10:1. It is found that volumetric entropy generation rate attains high values at passage inlet due to attainment of high temperature gradient in this region. Increasing rotation number and density ratio enhances volumetric entropy generation rate in the passage.
\end{abstract}

Keywords. Gas turbine; passage; entropy; rotation.

\section{Introduction}

Gas turbine blade cooling is necessary for efficient and long life operation. Increasing turbine inlet pressure increases significantly output power and thermal efficiency of a turbine. However, the thermal response of the material used in gas turbine limits the output power and efficiency of the operation. One of the solutions for improving turbine efficiency is to enhance gas turbine blade cooling. The cooling application requires efficient operation with proper selection of cooling parameters. Moreover, thermodynamic irreversibility associated with such a cooling system must be minimized for efficient processing. Consequently, investigation into thermodynamic irreversibility of the gas turbine blade cooling becomes essential.

Considerable research studies were carried out to examine gas turbine blade cooling. The influence of rotation on local heat transfer in a rectangular section duct was examined by Morris \& Ghavami-Nasr (1991). They showed that Coriolis induced secondary flows enhanced the local heat transfer over the trailing surface compared to stationary duct forced convection

*For correspondence 
situation. A numerical study of viscous flow in a rotating rectangular channel was carried out by Yang \& Fuchs (1993). They indicated that at low Reynolds numbers, a pair of stable vortex secondary flow and fully developed flow appeared in the channel even if the rotating rate was high. The effect of rotation on jet impingement heat transfer in smooth rectangular channels with four heated walls and radial outward cross flow was examined by Parson et al (1998). They showed that, as rotation number increases, the heat transfer coefficients for all walls in both channels decreased up to $20 \%$ below those results which corresponded to nonrotating conditions. Fully developed laminar flow and heat transfer in rectangular elliptical ducts rotating about a parallel axis was studied numerically by Mahadevappa et al (1996). They indicated that elliptical geometry was superior to others, which were frequently used as cooling channels in rotating machinery. Hydrodynamics and heat transfer in channels rotating about their axis were examined by Shevchuk \& Khalatov (1997). They indicated that rotational speed of the channel had a substantial effect on the characteristics of flow and heat transfer. Impingement cooling in rotating two-pass rectangular channels was studied by Akella \& Han (1998). They showed that rotation-induced Coriolis and centrifugal forces decrease the Nusselt number values within the range $20 \%$ and $25 \%$ in the impingement channels and turn regions, respectively. Flow and heat transfer characteristics in rotating two-pass rectangular channels were examined by Al-Qahtani et al (2002). They used Reynolds Stress Turbulence model and compared the predictions with experimental data for both stationary and rotating two-pass rectangular channels. Moreover, flow and heat transfer in rotating channels with $45^{\circ}$ rib turbulators were studied by Al-Qahtani et al (2003). They showed that for high rotation and high density ratio, the rotation-induced secondary flow overpowered the ribs induced secondary flow, which in turn changed significantly the heat transfer characteristics compared to the low rotation and low density case. Griffith et al (2002) investigated heat transfer in rotating rectangular cooling channels. They indicated that the orientation effect with the trailing-edge channel enhanced the heat transfer rates more than orthogonal channel.

Entropy generation in a thermal system can be considered as a quantitative measure of the thermodynamic irreversibility of the system. Consequently, system parameters minimizing entropy generation provides optimum conditions for efficient processing. Considerable research studies were carried out to examine entropy generation in flow systems in channels. Brizuela (1993) examined entropy generation and transport in the blade-tip layer of a radial impeller. He showed that most of entropy was generated at the fluid boundary and indicated that operating conditions minimizing entropy generation rate was existed. Entropy generation in open channel flow was investigated by Chiu \& Abidin Said (1995). He used constant entropy generation rate to determine channel section for various discharge conditions. Thermal optimization of channel flows with discrete heating sections was investigated by Furukawa \& Yang (2003). They showed that the broad spacing of channel for minimum entropy generation agreed well with that calculated by maximizing the thermal conductance induced by both heat transfer and viscous friction. Andreozzi et al (2006) studied entropy generation in natural convection in a symmetrically and uniformly heated vertical channel. They developed a correlation between global entropy generation rates, Rayleigh number and the aspect ratio of channel. Khan et al (2006) studied optimization of micro-channel heat sink using entropy generation minimization method. They showed that the effects of different design variables on the overall performance of micro-channel were significant. Yilbas (2001) examined entropy generation in concentric annuli with rotating outer cylinder. He showed that the efficient operation and design of bearing system could be possible with the analysis of entropy generation. 
In a thermal system, the source of entropy generation is due to heat transfer and the fluid friction. Entropy generation due to heat transfer increases with increasing the temperature gradient in the fluid while entropy generation due to fluid friction is associated with the rate of fluid strain in the flow system (Bejan 1995). In the case of convective cooling applications, heat absorbed by the working fluid is associated with the bulk mean temperature rise in the fluid; hence, uniformly increased bulk mean temperature of the working fluid is essential for effective cooling applications. However, high temperature gradient developed in the working fluid lowers the bulk mean temperature of the fluid while reducing the cooling effectiveness. In addition, high fluid strain developed in the flow results in excessive friction while increasing both the pressure drop and the required pumping power. Consequently, analysis of entropy generation due to; i) temperature gradient in the working fluid and ii) pressure drop is essential for the flow systems associated with the cooling applications. In the present study, rectangular passage resembling the turbine blade cooling passage is considered and entropy generation rate in the passage during cooling operation is examined. The leading and trailing wall (with respect to the direction of rotation) are assumed to be at constant temperature in the analysis. This is due to almost constant temperature hot gas flow at the outer surface of the blade. Reynolds stress turbulence model is used in the simulation to account for the turbulence. Control volume approach is introduced to discretize the governing equations of flow and heat transfer.

\section{Mathematical modelling}

\subsection{Governing equations of flow}

Continuity:

$$
\nabla \cdot\left(\begin{array}{c}
r \\
\rho \bar{u}
\end{array}\right)=0
$$

$x$-Momentum:

$$
\nabla \cdot\left(\begin{array}{c}
r \\
\rho \bar{u} \bar{u}
\end{array}\right)=-\frac{\partial p}{\partial x}-2 \rho \Omega w+\rho \Omega^{2} x+\nabla[\mu \operatorname{grad}(\bar{u})]+\nabla\left[-\rho \overline{u^{\prime} v^{\prime}}\right]
$$

$y$-Momentum:

$$
\nabla \cdot\left(\begin{array}{c}
r \\
\rho \bar{u} v
\end{array}\right)=-\frac{\partial p}{\partial y}+\nabla[\mu \operatorname{grad}(v)]+\nabla\left[-\rho \overline{v^{\prime} w^{\prime}}\right]
$$

z-Momentum:

$$
\nabla \cdot\left(\begin{array}{c}
r \\
\rho \bar{u} w
\end{array}\right)=-\frac{\partial p}{\partial z}+2 \rho \Omega \bar{u}+\rho \Omega^{2} z+\nabla[\mu \operatorname{grad}(w)]+\nabla\left[-\rho \overline{w^{\prime} u^{\prime}}\right]
$$

Energy:

$$
\nabla\left(\begin{array}{c}
r \\
\rho \bar{u}_{i} T
\end{array}\right)=\nabla\left[\left(\frac{\mu}{\operatorname{Pr}}\right) \operatorname{grad}(T)\right]+\nabla\left[-\rho \overline{u_{i}^{\prime} T}\right] .
$$

The second and third terms on the right hand sides of equation (2) and (4) occur due to the Coriolis and centrifugal forces, respectively. It is worth mentioning here that the governing equations are solved in rotating reference frame, where $u, v$ and $w$ represent relative velocity components. 


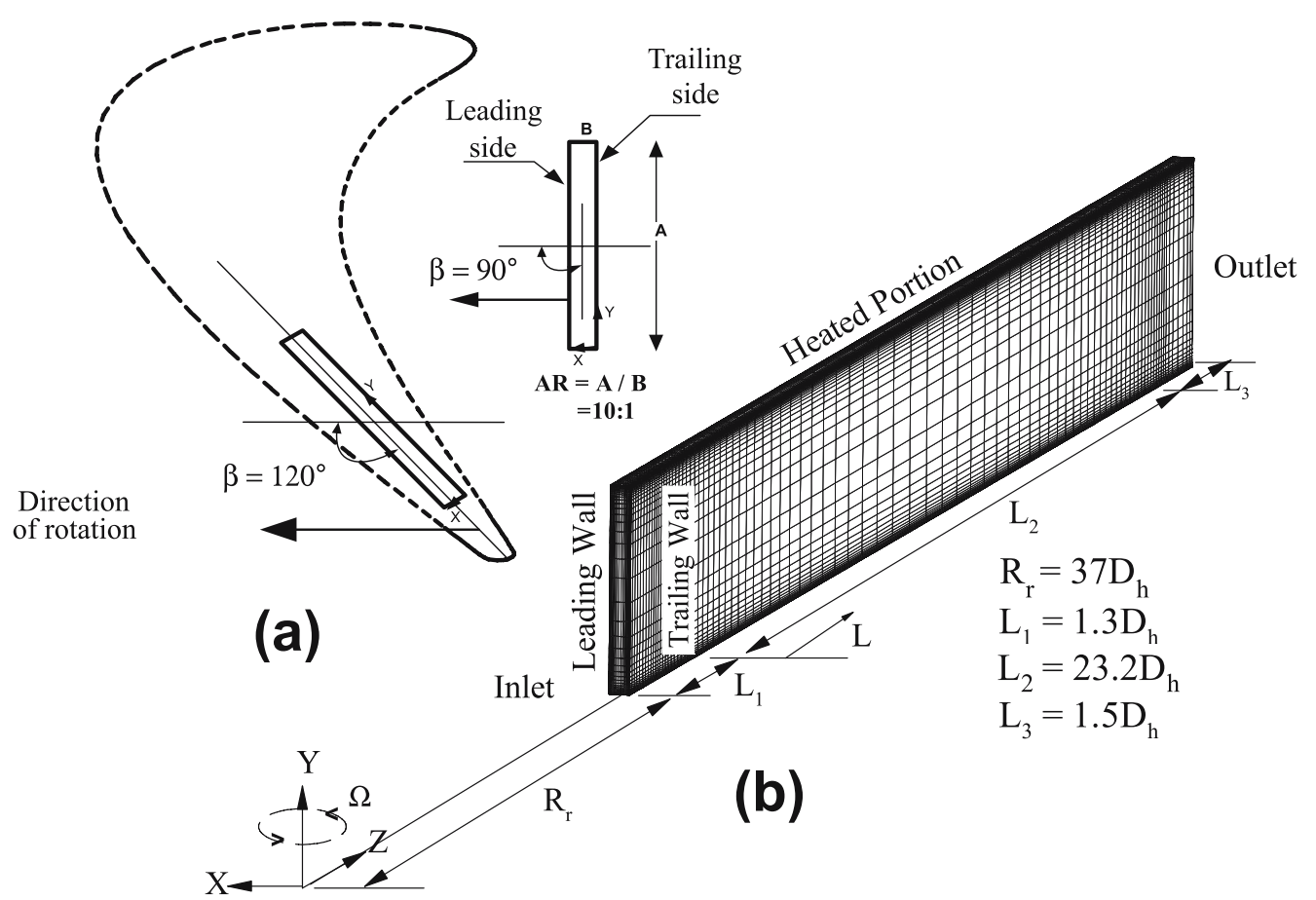

Figure 1. (a) Location of the tilted cooling channel in a turbine blade, (b) computational grid in the channel. $\beta$ is the angle of channel measured from direction of rotation, $R_{r}$ is the radial distance of the channel to axis of rotation.

\subsection{Turbulence modelling}

Since the cooling passage rotates while the flow passing through it (figure 1a), the turbulent kinetic energy generation and dissipation cannot be directly related to the mean flow properties. In this case, the $k-\varepsilon$ turbulence model does not correctly predict the turbulence quantities (Al-Qahtani et al 2002; Chen et al 2000). Therefore, Reynolds stress model is selected, which involves calculation of individual Reynolds stresses through solving the appropriate differential equations. The individual Reynolds stresses are then used to obtain closure of the Reynolds-averaged momentum equation. Following (David 2002) the model equations for the transport of the Reynolds stresses may be written as follows.

$$
C_{i j}=D_{i j}^{T}+D_{i j}^{L}+P_{i j}+G_{i j}+\phi_{i j}+\varepsilon_{i j}+F_{i j},
$$

where

$$
\begin{array}{rlrl}
C_{i j} & =\frac{\partial}{\partial x_{k}}\left(\rho \overline{u_{i} u_{i} u_{j}}\right) & \text { is the convective term, } \\
D_{i j}^{T}=-\frac{\partial}{\partial x_{k}}\left[\frac{\mu_{t}}{\sigma_{k}} \frac{\partial \overline{u_{i} u_{j}}}{\partial x_{k}}\right] & \text { is the turbulent diffusion term, } \\
D_{i j}^{L}=\frac{\partial}{\partial x_{k}}\left[\frac{\mu_{t}}{x_{k}}\left(\overline{u_{i} u_{j}}\right)\right] & \text { is the laminar diffusion term, }
\end{array}
$$




$$
\begin{aligned}
& P_{i j}=\left(\overline{u_{i} u_{j}} \frac{\partial U_{j}}{\partial x_{k}}\right)+\left(\overline{u_{i} u_{k}} \frac{\partial U_{i}}{\partial x_{k}}\right) \quad \text { is the stress production term, } \\
& G_{i j}=\beta \frac{\mu_{t}}{\operatorname{Pr}_{t}}\left(g_{i} \frac{\partial T}{\partial x_{j}}+g_{j} \frac{\partial T}{\partial x_{i}}\right) \quad \text { is the buoyancy production term, } \\
& \phi_{i j}=\phi_{i j, 1}+\phi_{i j, 2}+\phi_{i j}^{w} \quad \text { is the pressure strain term, } \\
& \phi_{i j, 1}=-C_{1} \rho \frac{\varepsilon}{k}\left(\overline{u_{i} u_{j}}-\frac{2}{3} \delta_{i j} k\right) \quad \text { is the slow pressure strain term, } \\
& \phi_{i j, 2}=-C_{2}\left[\left(P_{i j}+F_{i j}+G_{i j}-C_{i j}\right)-\frac{1}{3} \delta_{i j}\left[P_{k k}+G_{k k}-C_{k k}\right]\right] \\
& \phi_{i j}^{w}= C_{1}^{\prime} \frac{\varepsilon}{k}\left(\overline{u_{k} u_{m}} n_{k} n_{m} \delta_{i j}-\frac{3}{2} \overline{u_{i} u_{k}} n_{j} n_{k}-\frac{3}{2} \overline{u_{j} u_{k}} n_{i} n_{k}\right) \frac{k^{3 / 2}}{C_{1} \varepsilon d} \\
&+C_{2}^{\prime}\left(\phi_{k m, 2} n_{k} n_{m} \delta_{i j}-\frac{3}{2} \phi_{i k, 2} n_{j} n_{k}-\frac{3}{2} \phi_{j k, 2} n_{i} n_{k}\right) \frac{k^{3 / 2}}{C_{1} \varepsilon d}
\end{aligned}
$$

is the wall reflection term,

$d$ is the normal distance to the wall. $n_{k}$ and $x_{k}$ component of the unit normal to the wall.

$$
\begin{array}{ll}
\varepsilon_{i j}=-2 \mu \frac{\overline{\partial u_{i} \partial u_{i}}}{\partial x_{k} \partial x_{k}} & \text { is the dissipation term, } \\
F_{i j}=-2 \rho \Omega_{k}\left(\overline{u_{j} u_{m}} \varepsilon_{i k m}+\overline{u_{i} u_{m}} \varepsilon_{i k m}\right) & \text { is the rotation term. }
\end{array}
$$

Where $C_{1}=1.8, C_{2}=0.6, C_{1}^{\prime}=0.5, C_{2}^{\prime}=0.3, C_{l}=\frac{C_{l}^{3 / 2}}{k}, C_{\mu}=0.09 \& k=0.41$ are the suggested values by Furukawa \& Yang (2003).

To close the above system of equations, expressions need to be provided for the turbulence viscosity $\mu_{t}$ and the turbulence Prandtl number $\operatorname{Pr}_{t}$. The turbulent viscosity is obtained using Reynolds Stress Model where $k$ and $\varepsilon$ represent the turbulent kinetic energy and rate of dissipation of turbulent kinetic energy respectively.

Turbulent viscosity is expressed involving $k$ and $\varepsilon$ as: (David 2002)

$$
\mu_{t}=\rho C_{\mu} \frac{k^{2}}{\varepsilon}
$$

$k$ and $\varepsilon$ are solved from the following two transport equations,

$$
\rho \frac{D k}{D t}=\frac{\partial}{\partial x_{j}}\left[\left(\mu+\frac{\mu_{t}}{\sigma_{k}}\right) \frac{\partial k}{\partial x_{j}}\right]+\frac{1}{2}\left(P_{i i}+G_{i i}\right)-\rho \varepsilon\left(1+2 M_{t}^{2}\right)
$$

where $\sigma_{k}=0.82 M_{t}=\sqrt{\frac{k}{a^{2}}}$ where $M_{t}$ is Mach number, and $a(\equiv \sqrt{\gamma R T})$

$$
\rho \frac{D \varepsilon}{D t}=\frac{\partial}{\partial x_{j}}\left[\left(\mu+\frac{\mu_{t}}{\sigma_{\varepsilon}}\right) \frac{\partial \varepsilon}{\partial x_{j}}\right]+C_{\varepsilon 1} \frac{1}{2}\left(P_{i i}+C_{\varepsilon 3} G_{i i}\right) \frac{\varepsilon}{k}-C_{\varepsilon 2} \rho \frac{\varepsilon^{2}}{k} .
$$


Where $\sigma_{\varepsilon}=1.0, C_{\varepsilon 1}=1.44, C_{\varepsilon 2}=1.92$, (are the suggested values by Fluent (2003) and $C_{\varepsilon 3}=\tanh |v / u|$ where $v$ is the component of the flow velocity parallel to the gravitation vector and $u$ is the component of the flow velocity perpendicular to the gravitational vector.

\subsection{Entropy equation}

After considering time averaged mean velocity and temperature, entropy generation for threedimensional fluid flow and heat transfer is given by Bejan (1995):

$$
\begin{aligned}
S_{\text {gen,cond }}^{\prime \prime \prime}= & \frac{k_{\text {eff }}}{T_{I}^{2}}\left[\left(\frac{\partial T}{\partial x}\right)^{2}+\left(\frac{\partial T}{\partial y}\right)^{2}+\left(\frac{\partial T}{\partial z}\right)^{2}\right] \\
S_{\text {gen,frict }}^{\prime \prime \prime}= & \frac{\mu_{e f f}}{T_{I}}\left[2\left(\left(\frac{\partial u}{\partial x}\right)^{2}+\left(\frac{\partial v}{\partial y}\right)^{2}+\left(\frac{\partial w}{\partial z}\right)^{2}\right)\right. \\
& \left.+\left(\frac{\partial u}{\partial y}+\frac{\partial v}{\partial x}\right)^{2}+\left(\frac{\partial u}{\partial z}+\frac{\partial w}{\partial x}\right)^{2}+\left(\frac{\partial v}{\partial z}+\frac{\partial w}{\partial y}\right)^{2}\right],
\end{aligned}
$$

where $k_{\text {eff }}$ is the effective thermal conductivity and $k_{\text {eff }}=k_{l}+k_{t}, k_{l}$ being bulk thermal conductivity and $k_{t}$ is turbulence contribution. Similarly, $\mu_{e f f}$ is the effective viscosity and $\mu_{e f f}=\mu_{l}+\mu_{t}, \mu_{l}$ being bulk viscosity and $\mu_{t}$ is turbulence contribution.

Following Yilbas et al (1999), the total volumetric entropy generation after being normalized can be written as:

$$
S_{\text {gen }}^{\prime \prime \prime} \frac{D_{h}^{2}}{k}=\sigma^{2}\left(\nabla T^{*}\right)^{2}+\operatorname{Pr} E c \sigma\left(\Phi^{*}\right) .
$$

Where $T^{*}=\frac{T}{\left(\dot{q} / \rho C_{p} u_{f}\right)} ; \sigma=\frac{\left(\dot{q} / \rho C_{p} u_{f}\right)}{T}$ and Pr is Prandtl number; $\operatorname{Pr}=\mu C_{p} / k$ and $E c$ is Eckert number $E c=W_{i}^{2} /\left(C_{p}\left(\dot{q} / \rho C_{p} u_{f}\right)\right)$. The span-wise averaged volumetric entropy generation rate is determined from integrating the volumetric entropy generation rate along the span length and dividing the integrated value to the span length.

\section{Boundary conditions}

At the inlet $(z=0)$, a uniform velocity profile will be used for the $w$-component while the $u$, $v$-components assumed to be zero. The turbulence intensity is set $4 \%$ at the inlet (Al-Qahtani et al 2002; Chen et al 2000). Reynolds stresses are assumed to be isotropic at the inlet. Normal stresses $\overline{u^{\prime} u^{\prime}}, \overline{v^{\prime} v^{\prime}}$ and $\overline{w^{\prime} w^{\prime}}$ are set to 1 , and $\overline{u^{\prime} v^{\prime}}, \overline{u^{\prime} w^{\prime}}, \overline{v^{\prime} w^{\prime}}$ are set to zero (Fluent 2003). Since we are using ideal gas law to incorporate the density variation with temperature, the code does not permit use of zero-gradient boundary conditions for the flow variables at the exit of the passage. The buoyancy effect is expected to be significant at high rotation number, Ro, and high density ratio, $\Delta \rho / \rho$, so, we may expect some flow reversal to occur near the outlet of the passage. Due to this reason pressure is fixed to atmospheric pressure at the exit. Reynolds number based on passage hydraulic diameter is fixed to 30000 . The value of Reynolds number is selected due to the practical cooling applications (Al-Qahtani et al 2002; Willet \& Bergles 2001). At the walls, the no-slip condition is used. The coolant fluid 
at the inlet of the passage is at uniform temperature $T=T_{i}$. The top and bottom walls are unheated and thermally insulated in the experiment of Willet \& Bergles (2001) so they were modelled with zero heat flux boundary condition. The leading and trailing walls are modelled with constant temperature.

\section{Numerical solution}

The finite volume code, FLUENT is used to predict flow and heat transfer characteristics. The grid was generated iteratively using Gambit software. Figure $1 \mathrm{~b}$ shows the computational grid. The block was divided into three zones, to have better distribution of nodes in the critical regions. Reynolds stress model in conjunction with two-layer approach is used. The minimum grid spacing (first node from the wall) in the near-wall region is maintained at reasonable distance, which corresponds to a wall coordinate $y^{+}$of the order of 1 . The number of grid points in the stream wise direction from inlet to outlet is 88 while in the cross-stream plane is $41 \times 61$. The grid was made dense in the region of high gradients, such as at the near-walls, inlet and at the two junctions of heated and unheated section in order to obtain accurate results. The number of grid points and their distribution in the present smooth duct were obtained based on grid-refinement test. First order upwind scheme is used to compute velocity field, and SIMPLE algorithm is used to couple pressure with velocity field. Convergence criteria were set to $10^{-5}$ for all the flow variables.

To choose a suitable computational grid, a grid independence test must be performed, to ensure grid independence. The solutions were computed using different grid sizes. The tested grid sizes are $31 \times 51 \times 88,41 \times 61 \times 88$, and $57 \times 71 \times 88$. From now onwards, these grids will be referred to as Grid-1, Grid-2, and Grid-3, respectively. The parameters used to check the grid independency are axial velocity and temperature. Grid refinement tests in the cross stream direction were carried out and it was found that the accuracy of the solution on Grid-2 $(41 \times 61 \times 88)$ is over $99 \%$, which was considered to be satisfactory. To check the grid sensitivity in stream-wise direction, 16 nodes were increased in $z$-direction. No appreciable difference in temperature distribution was found (less than $0.01 \%$ ). Thus Grid-2 $(41 \times 61 \times 88)$ was used for the remaining of the computation. The corresponding CPU time on Xeon $3.06 \mathrm{Ghz} / 2 \mathrm{~Gb}$ RAM computer is about $16 \mathrm{~h}$ for convergence, and typically it takes 1508 iterations to reach convergence.

\section{Validation}

To validate the results, the simulations were repeated for the same conditions of rotation number, Ro, and density ratio, $\Delta \rho / \rho$, reported in the open literature. The Nusselt number ratios at the leading and trailing wall were computed and compared with their contour parts presented in the previous study (Willet \& Bergles 2001). The results are shown in figure 2. It can be seen that the computed Nusselt number ratios are very close to the experiment data (Willet \& Bergles 2001) for the leading and the trailing walls. However, small differences between both results can be attributed to the one or all of the followings: (i) the uncertainty involved in experimental data was reported as \pm 11 percent, and (ii) the truncation errors involved in the numerical simulations. Nevertheless, the differences between both results are very small. 


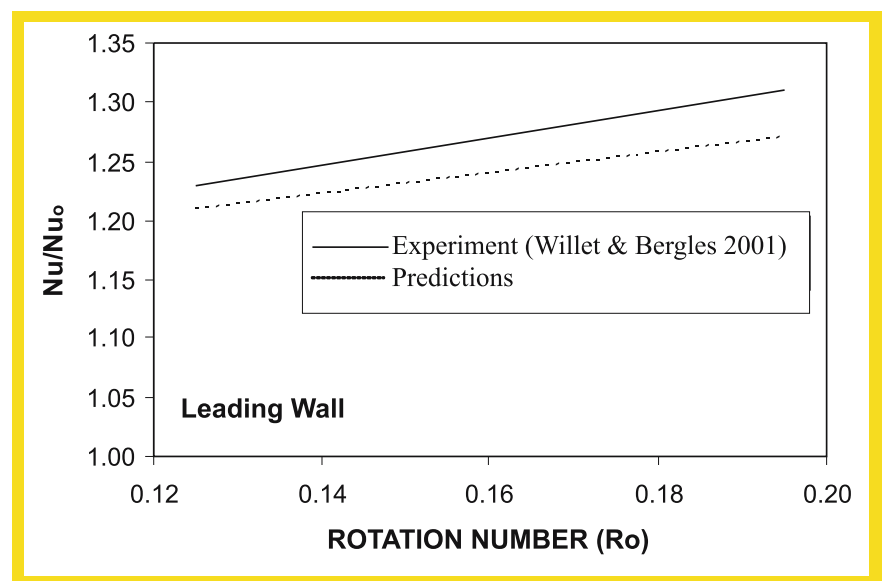

(a)

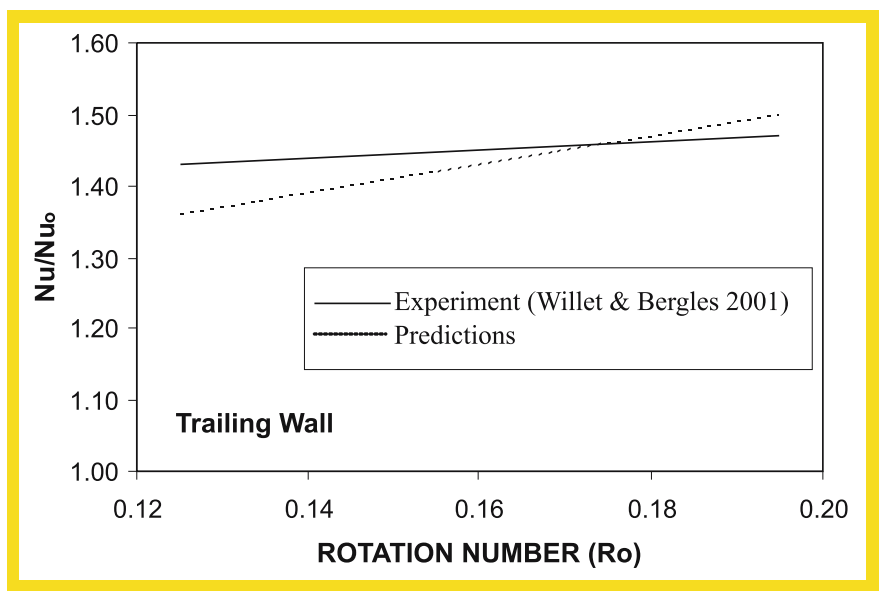

(b)

Figure 2. Averaged Nusselt number comparison due to present study and the previous experiment (Willet \& Bergles 2001): (a) leading wall; (b) trailing wall.

\section{Results and discussions}

Flow into a passage resembling a turbine blade cooling passage with rotation is considered and entropy generation in the flow field is examined. In the entropy analysis, two rotational speeds and three density ratios are accommodated. The passage aspect ratio is kept 10:1 and $\operatorname{Re}=3 \times 10^{4}$. Constant temperature boundaries are assumed at leading and trailing walls. Table 1 gives the fluid properties used in the simulations.

Span-wise view is shown in figure 3 . Figure 4 shows span-wise averaged volumetric entropy generation rate at three planes in the passage for two rotational speeds and different density ratios. Entropy generation rate in the region close to the leading wall (figure $4 \mathrm{a}$ ) is high in the entrance region of the passage and it decreases gradually with increasing span-wise distance towards the passage exit. The attainment of high entropy generation rate is because of the low temperature working fluid entering the passage. This in turn, increases the temperature 
Table 1. Fluid properties.

\begin{tabular}{lccc}
\hline Fluid & Viscosity $(\mathrm{kg} / \mathrm{m}-\mathrm{s})$ & Thermal conductivity $(\mathrm{W} / \mathrm{m}-\mathrm{K})$ & Specific heat capacity $(\mathrm{kJ} / \mathrm{kg}-\mathrm{K})$ \\
\hline R134a & $1.189810^{-5}$ & 0.013538 & 854 \\
\hline
\end{tabular}

gradient and the rate of fluid strain due to mixing in near downstream. Consequently, volumetric entropy generation rate increases in this region. As the flow passes towards the passage exit, it is heated by the leading and trailing walls, since the leading and trailing walls are at higher temperature than that of the fluid. Therefore, the gradual increase in fluid temperature results in the gradual decay of temperature gradient in the fluid. This causes gradual decay of entropy generation rate towards the passage exit. Moreover, entropy generation rate attains high values when density ratio increases. It should be noted that density ratio is associated with temperature difference between wall and fluid $\left(\Delta \rho / \rho=\left(T_{w}-T_{i}\right) / T_{w}\right)$. In this case, increasing density means lowering fluid temperature, since wall temperature remains constant. In the case of trailing wall region (figure $4 b$ ), volumetric entropy generation rate attains high values in the passage inlet and it decays sharply as the working fluid progresses in the passage. The decay in volumetric entropy generation rate becomes monotonic as the distance from the inlet to passage exit increase. The sharp decay of volumetric entropy generation in the inlet region of the passage is associated with flow mixing in this region. Moreover, the magnitude of volumetric entropy generation rate is similar to that obtained in the leading wall region. It should be noted that leading and trailing walls of the passage are kept at constant temperature. In the case of mid-plane (midway between leading and trailing walls), volumetric entropy generation rate differs than its counter parts corresponding to leading and trailing wall regions. Increasing volumetric entropy generation in the downstream of the mid-plane is because of high leading and trailing wall temperatures, which in turn, enhances conduction and convection heat transfer from walls to the working fluid. Therefore, fluid temperature becomes high in the region close to the walls while it remains low in the mid-plane. This causes attainment of high temperature gradient in the region of mid-plane. In addition, thickening of thermal boundary and mixing of natural and forced convection currents in mid-plane enhances temperature gradient in this region. Consequently, entropy generation rate increases.

Figure 5 shows volumetric entropy generation rate along the span-wise direction at there planes in the passage for the three density ratios while $R o=0.25$ and $\beta=120^{\circ}$. It should be noted that Ro is the rotation number and $\beta$ is the angle of passage orientation measured as

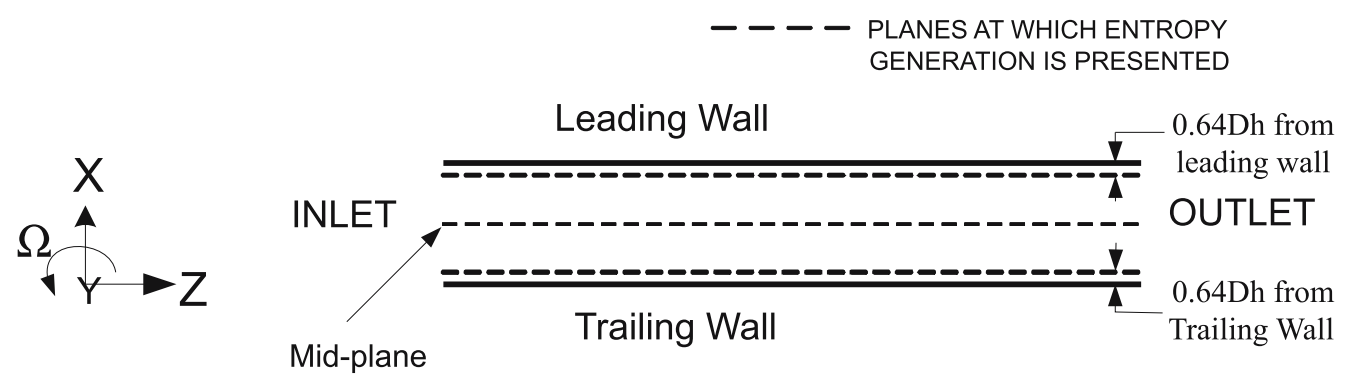

Figure 3. Location of planes in the channel. 

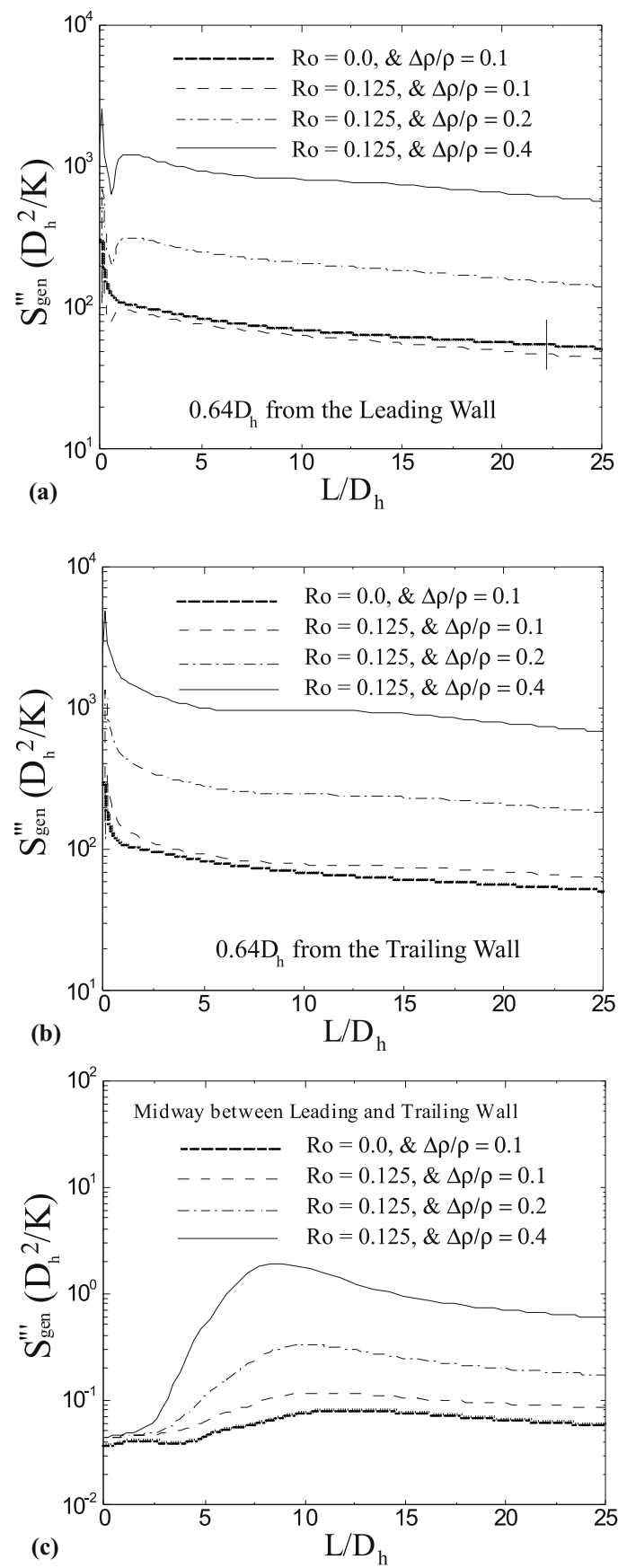

Figure 4. Span-wise averaged dimensionless volumetric entropy generation, $R o=0.0$ to $0 \cdot 125$ and $\beta=90^{\circ}$ : (a) leading wall; (b) trailing wall; (c) mid-plane.

reference to the direction of rotation. Volumetric entropy generation rate is high in the passage inlet and as the span-wise distance increases towards the passage exit it remains almost the same for each density ratio. However, increasing density ratio reduces fluid temperature at passage inlet, which in turn results in attainment of high temperature gradient in the passage. This enhances entropy generation rate in all locations in the passage. This situation is also 

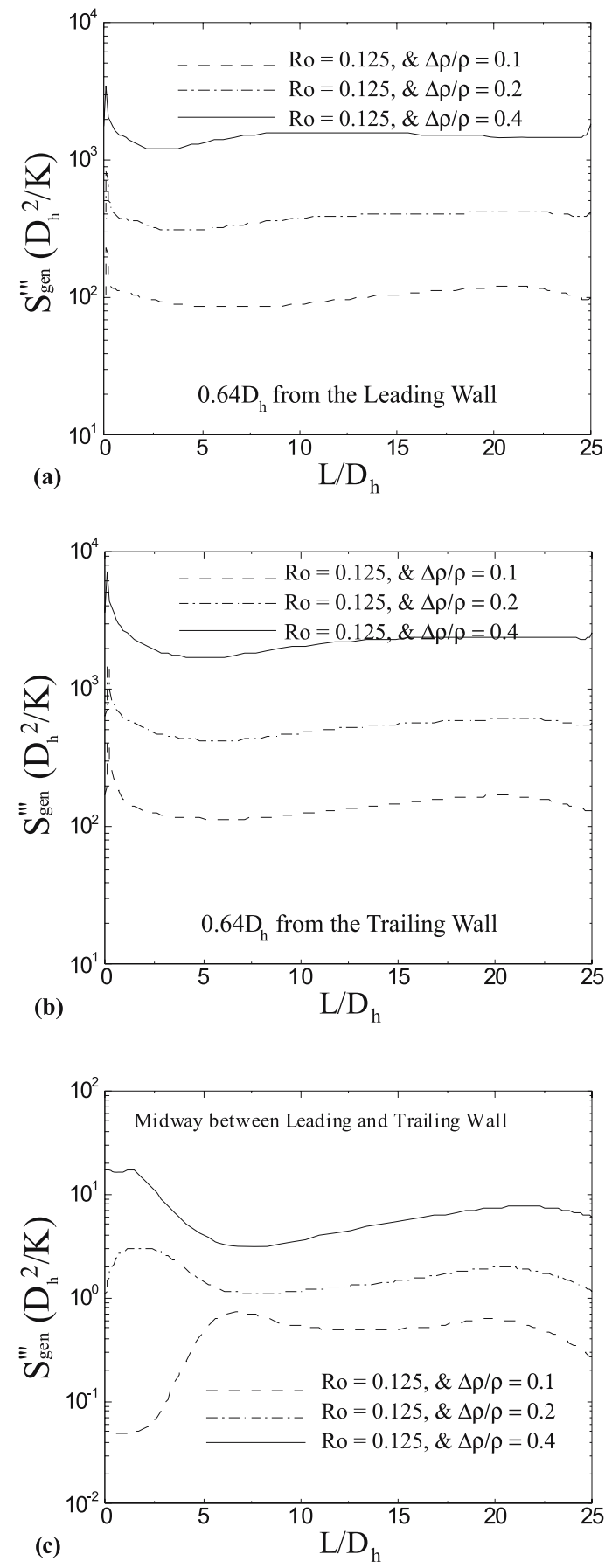

Figure 5. Span-wise averaged dimensionless volumetric entropy generation, $R o=0.25$ and $\beta=120^{\circ}$ : (a) leading wall; (b) trailing wall; (c) mid-plane.

seen in the regions close to trailing wall. However, variation in volumetric entropy generation rate occurs along the span-wise direction in the region at midway between leading and trailing walls. This is true for all density ratios. The variation of entropy generation rate in this region 

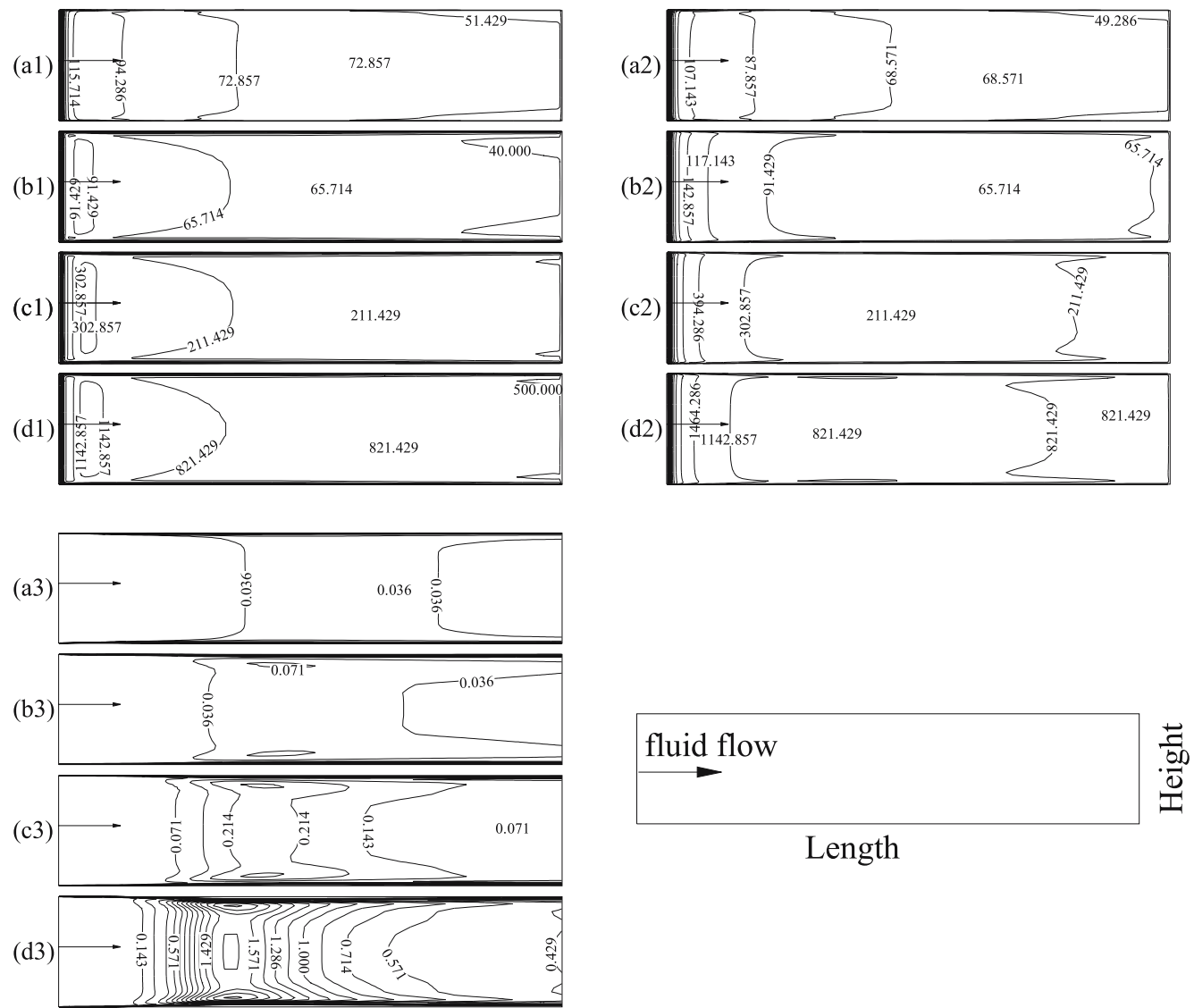

Figure 6. Local dimensionless volumetric entropy generation, $R o=0.0$ and $0 \cdot 125, \beta=90^{\circ}$ : (1) leading wall; (2) trailing wall; (3) mid-plane; (a) $R o=0 \cdot 0$ and $\Delta \rho / \rho=0 \cdot 1$, (b) $R o=0 \cdot 125$ and $\Delta \rho / \rho=0 \cdot 1$, (c) $R o=0.125$ and $\Delta \rho / \rho=0 \cdot 2$, (d) $R o=0.125$ and $\Delta \rho / \rho=0.4$.

is associated with the heat transfer rates from leading and trailing walls to the working fluid in the passage. Consequently, mixing of forced convection and natural convection currents and conduction heating in the working fluid result in variation of temperature gradient along the span-wise direction at mid-plane in the passage. This is more pronounced as the density ratio increases. When comparing figures 4 and 5 , it is observed that reducing rotation number $(R o)$ while increasing passage orientation $(\beta)$ modifies entropy generation rate in the region of leading and trailing walls. However, this effect is less pronounced at the mid-plane.

Figure 6 shows contours of volumetric entropy generation rates for different rotation numbers while $\beta=90^{\circ}$. Increasing rotation number changes entropy distribution in the region close to all planes. This change becomes significant once the density ratio increases. This is because of the Coriolis effect due to rotational motion of the passage. Moreover, flow mixing in the passage enhances the frictional losses and improves temperature uniformity in the passage, in particular in the region close to mid-plane. However, the thermal boundary layer developed within the vicinity of leading and trailing walls causes high temperature gradient in the region next to the thermal boundary layers. Once the density ratio increases, temperature gradient becomes large and entropy generation rate due to heat transfer increases significantly 

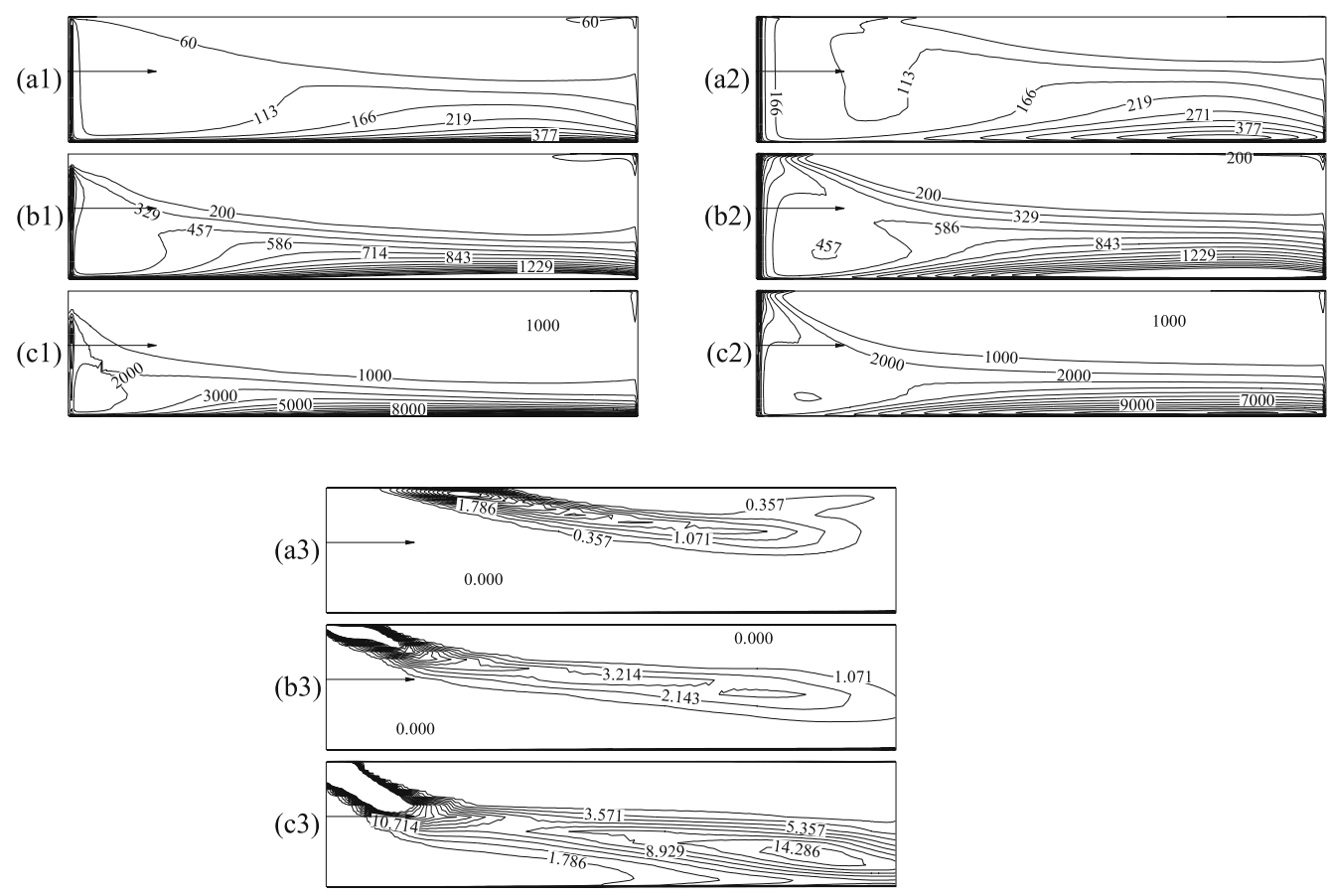

Figure 7. Local dimensionless volumetric entropy generation, $R o=0 \cdot 25$ and $\beta=120^{\circ}$ : (1) leading wall; (2) trailing wall; (3) mid-plane; (a) $R o=0.25$ and $\Delta \rho / \rho=0.1$; (b) $R o=0.25$ and $\Delta \rho / \rho=0.2$; (c) $R o=0.25$ and $\Delta \rho / \rho=0.4$.

in this region. This causes local variation of entropy generation rate across the passage as well as along the planes next to the leading and trailing walls.

Figure 7 shows contours of entropy generation rate in the planes of leading wall region, trailing wall region, and midway in the passage for different rotation numbers and density ratios while $\beta=120^{\circ}$. Variation in entropy generation with increasing rotational speed and density ratio is evident. However, span-wise variation in entropy is not as significant as the case occurred for $\beta=90^{\circ}$. This can be attributed to temperature distribution and viscous dissipation in the passage. Therefore, temperature gradient does not vary as much as that of $\beta=90^{\circ}$ and viscous dissipation within the passage does not change drastically for $\beta=120^{\circ}$. This indicates that Coriolis effect on the flow field is suppressed once the orientation angle of $\beta$ changes from $90^{\circ}$ to $120^{\circ}$. This situation is also observed from a previous study (Al-Qahtani et al 2002). However, flow mixing at high density ratios increases viscous dissipation and temperature gradient in the passage. This situation is more pronounced in the regions close to leading and trailing walls. Consequently, the magnitude of volumetric entropy generation rate increases significantly for high density ratios. It should be noted that increasing density ratio lowers fluid temperature at passage inlet, which enhances temperature gradient in the passage.

Figure 8 shows the ratio of volumetric entropy generation rate along the span-wise direction for two $\beta$ values. The small variation in entropy ratio at passage inlet is associated with the attainment of high temperature gradient in the inlet region of the passage. As the spanwise distance increases towards the passage exit, heat transfer from leading and trailing walls suppresses temperature gradient variation in the passage. This, in turn, results in steady 


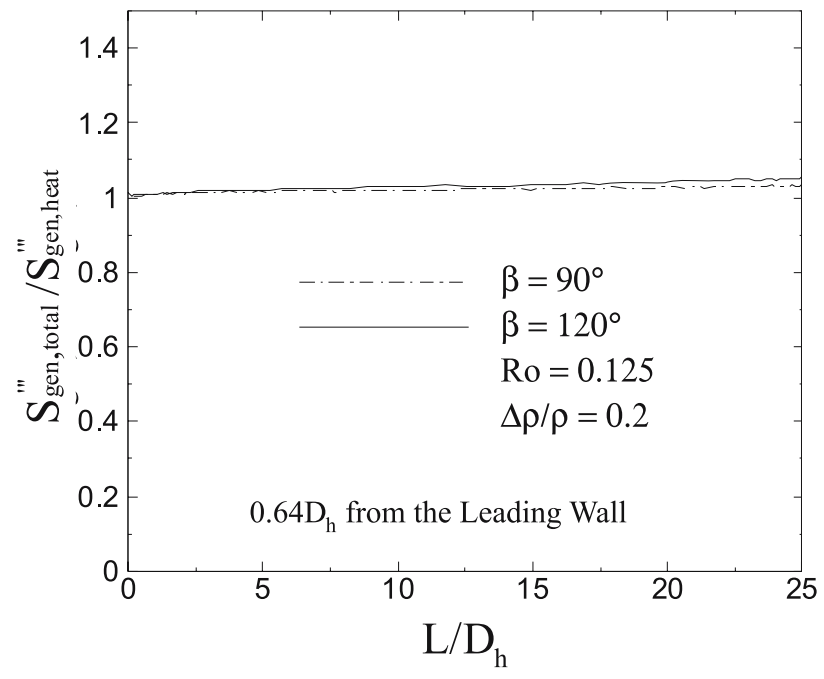

Figure 8. Span-wise total entropy ratio on a plane close to leading wall. $R o=0.125$ and $\Delta \rho / \rho=$ 0.2 .

variation of entropy ratio. Moreover, changing the orientation of the passage from $90^{\circ}$ to $120^{\circ}$, entropy ratio does not change considerably. In this case, mixing in the passage is partially suppressed and temperature gradient becomes almost the same for both $\beta$ values. Consequently, entropy ratio remains almost 1 . This situation is true for all locations in the span-wise direction.

\section{Conclusion}

Flow in a passage resembling gas turbine cooling passage is considered and entropy generation in the passage is examined for various rotation numbers and density ratios and two passage orientations. A numerical scheme using a control volume approach is introduced to discretize the governing equations of flow and entropy. The Reynolds stress turbulence model is accommodated to account for the turbulence in the passage. It is found that volumetric entropy generation rate attains high values in the entry region of the passage because of the high viscous dissipation and the attainment of high temperature gradient in this region. This is more pronounced with increasing density ratio due to the low fluid temperature at passage inlet. This also enhances temperature gradient in this region. Moreover, volumetric entropy generation rate increases with increasing rotation number. In this case, Coriolis effect enhances temperature gradient in the region next to leading and trailing walls. Increasing $\beta$ from $90^{\circ}$ to $120^{\circ}$ reduces volumetric entropy generation rate in the passage. The change in the orientation of the passage is associated with the Coriolis effect, which is suppressed with increasing $\beta$. Slightly increasing trend is observed for entropy ratio along the span-wise direction towards the passage exit. However, the difference in entropy ratio due to $\beta=90^{\circ}$ and $\beta=120^{\circ}$ is considerably small.

\section{List of symbols}

$C_{p} \quad$ Specific heat, $\mathrm{kJkg}^{-1} \mathrm{~K}^{-1}$

$D_{h} \quad$ Hydraulic diameter, $\mathrm{m}$ 


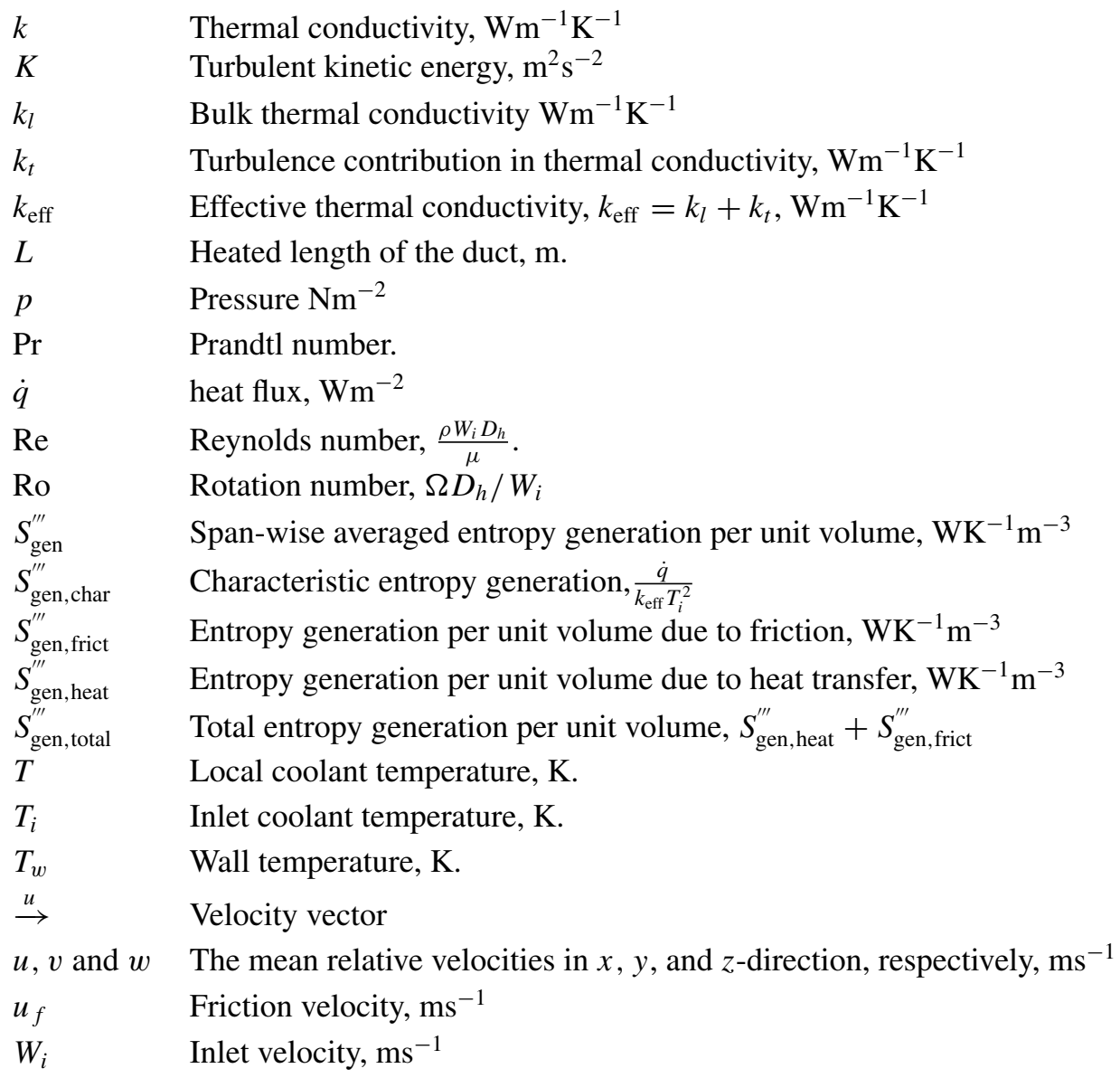

\section{Greek symbols}

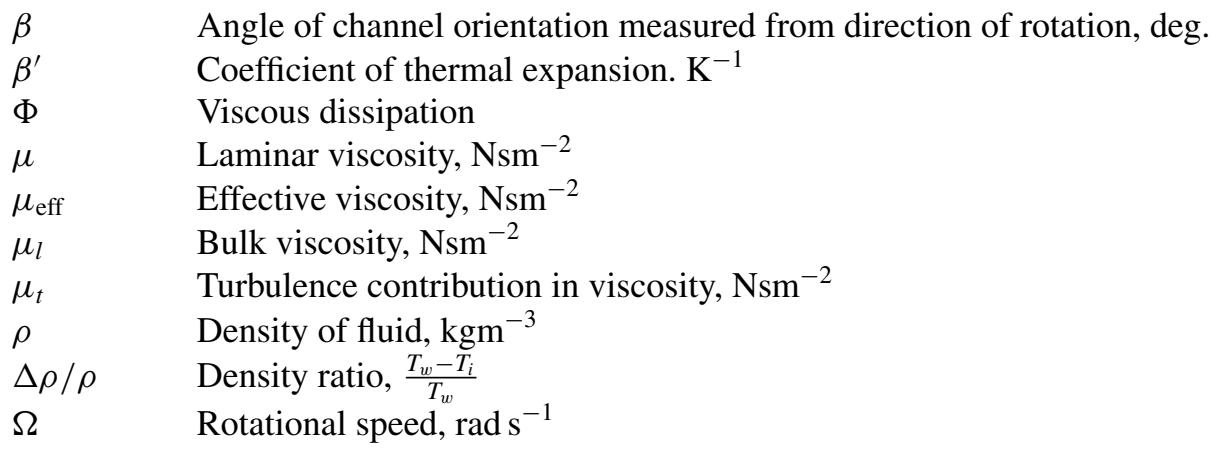

\section{References}

Akella K V, Han J C 1998 Impingement cooling in rotating two-pass rectangular channels. J. Thermophysics and Heat Transfer 12(4): 582-88 
Al-Qahtani M, Jang Y J, Chen H C, Han J C 2002 Flow and heat transfer in rotating two-pass rectangular channels $(A R=2)$ by Reynolds stress turbulence model. Int. J. Heat and Mass Transfer 45(9): 1823-38

Al-Qahtani M, Chen H C, Han J C 2003 A numerical study of flow and heat transfer in rotating rectangular channels $(A R=4)$ with $45^{\circ}$ rib turbulators by Reynolds stress turbulent model. J. Heat Transfer 125(1): 19-26

Andreozzi A, Auletta A, Manca O 2006 Entropy generation in natural convection in a symmetrically and uniformly heated vertical channel. Int. J. Heat and Mass Transfer 49(17-18): 3221-28

Bejan A 1995 Entropy Generation Minimization (New York: CRC Press), chapter 2

Brizuela E A 1993 Numerical modelling of entropy generation and transport in the blade-tip gap layer of radial impeller. ASME, Int. Gas Turbine and Aeroengine Congress and Exposition 93-GT-81

Chen H C, Jang Y J, Han J C 2000 Computation of heat transfer in rotating two-pass square channel by a second-moment closure model. Int. J. Heat and Mass Transfer 43(9): 1603-16

Chiu, C, Abidin Said C A 1995 Maximum and mean velocity and entropy in open channel. J. Hydraulic Eng. 121(1): 26-35

David C W 2002 Turbulence modelling for CFD (New York: McGraw-Hill), chapter 2

FLUENT 6.1 2003 User's Guide (Lebanon: Fluent Inc), chapter 9

Furukawa T, Yang W 2003 Thermal optimization of channel flow with discrete heating sections. J. Non-Equilibrium Thermodynamics 28(4): 299-310

Griffith T S, Al-Hadhrami L, Han J C 2002 Heat transfer in rotating rectangular cooling channel $(A R=4)$ with dimples, ASME, Turbo Expo GT-2002-30220

Khan W A, Yovanovich M M, Culham J R 2006 Optimization of microchannel heat sinks using entropy generation minimization method. Annual IEEE Semiconductor Thermal Measurement and Management Symposium 78-86

Mahadevappa M, Rao V R, Sastri V M K 1996 Numerical study of laminar fully developed fluid flow and heat transfer in rectangular and elliptic ducts rotating about a parallel axis. Int. J. Heat and Mass Transfer 39(4): 867-75

Morris W D, Gavami-Nasr 1991 Heat transfer measurements in rectangular channels with orthogonal mode rotation. J. Turbomachinery 113(3): 339-45

Parson J A, Han J C, Lee C P 1998 Rotation effect on jet impingement heat transfer in smooth rectangular channels with four heated walls and radially outward cross flow. J. Turbomachinery 120: 79-85

Shevchuk I V, Khalatov A A 1997 Heat transfer and hydrodynamics in channels rotating about their axis. Inzhenerno-Fizicheskii Zhurnal 70(3): 514-28

Willet F T, Bergles A E 2001 Heat Transfer in Rotating Narrow Rectangular Ducts With Heated Sides Oriented at $60^{\circ}$ to the r-z Plane. J. Turbomachinery 123: 288-95

Yang Y, Fuchs L 1993 Numerical study of viscous flow in a rotating rectangular channel. Int. J. Eng. Sci. 31(6): 873-881

Yilbas B S 2001 Entropy analysis of concentric annuli with rotating outer cylinder. Exergy Int. J. 1: 60-66

Yilbas B S, Shuja S Z, Budair M O 1999 Second analysis of a swirling flow in circular duct with restriction. Int. J. Heat and Mass Transfer 42: 4027-41 